\title{
VARIABILITÉ TEMPORELLE DES PEUPLEMENTS PISCICOLES DANS LA SECTION MÉDIANE DU BAS-RHÔNE.
}

\author{
G. CARREL (1), D. PONT (2), B. RIVIER (1)
}

(1) CEMAGREF, Division Hydrobiologie, BP 31, Le Tholonet, 13612 Aix-en-Provence Cedex 1, France.

(2) CNRS, URA 1974, équipe DESMID, Laboratoire d'Ecologie des Systèmes Fluviaux, 1 rue Parmentier, 13200 Arles, France.

\section{RÉSUMÉ}

Le suivi du peuplement piscicole du Bas-Rhône (aménagements de Montélimar et de Donzère-Mondragon), réalisé entre 1983 et 1994, permet d'aborder la variabilité interannuelle au sein de l'ichtyocénose.

L'étude porte sur 3 stations représentatives des configurations artificielles du cours principal : le chenal lotique, la retenue et le canal.

Parmi 33 espèces connues à l'échelle de ce secteur, 29 ont été capturées au printemps dans ces 3 stations. Cinq espèces euryoeciques (Rutilus rutilus, Alburnus alburnus, Leuciscus cephalus, Anguilla anguilla et Lepomis gibbosus), dont les occurrences sont supérieures à $60 \%$, représentent $92 \%$ de l'effectif total.

Au cours de cette décennie, 3 espèces sont apparues sur l'axe fluvial: Silurus glanis (1987), Pseudorasbora pana (1989) et Carassius auratus (1990).

Indépendamment des perturbations anthropiques, la variabilité interannuelle du nombre d'espèces et des effectifs est essentiellement déterminée par:

- la succession d'événements hydroclimatiques marqués (déficit hydrique des années 1989 à 1993) et crues exceptionnelles (octobre 1993 et janvier 1994),

- les stratégies écologiques des espèces vis-à-vis des contraintes abiotiques et biotiques.

Ces résultats mettent l'accent sur la nécessité de longues séries de relevés pour appréhender la variabilité des ichtyocénoses des grands cours d'eau et évaluer l'impact des aménagements.

Mots-clés : ichtyocénose, variabilité interannuelle, Bas-Rhône, chenal aménagé.

\section{TEMPORAL VARIABILITY OF FISH COMMUNITIES IN THE MEDIAN PART OF THE LOWER RHÔNE RIVER.}

\section{SUMMARY}

A study in the regulated part of the Lower Rhône (hydropower dams of Montélimar and Donzère-Mondragon) allowed us to identify the between year variability of fish assemblages from 1983 to 1994.

Observations concerned three stations representative of the artificially modified main channel : dyked lotic channel, storage reservoir and diversion canal.

Twenty-nine species among the thirty-three known from this part of the river were captured in the three stations during spring.

Five euryoecious species (Rutilus rutilus, Alburnus alburnus, Leuciscus cephalus, Anguilla anguilla and Lepomis gibbosus) with high occurrences in samples (more than $60 \%$ ) represented $92 \%$ of catches.

During the last decade, three new species appeared in the Lower Rhône: Silurus glanis (1987), Pseudorasbora parva (1989) and Carassius auratus (1990). 
Apart from anthropogenic disturbances, interannual variability in species richness and fish abundances were mainly determined by:

- successive and significant hydroclimatic events (hydrological deficit from 1989 to 1993) and high floods (October 1993 and January 1994),

- ecological strategies of species facing abiotic and biotic constraints.

The results prove the interest of long-term studies to appreciate variability in large river fish assemblages and the impact of river equipment.

Key-words : river fishes, community, temporal variability, regulated river, Lower Rhône River.

\section{INTRODUCTION}

Postérieurement à la cartographie piscicole du Rhône avant aménagement (KREITMANN, 1932 ; LEGER, 1945), une étude globale des peuplements ne sera réalisée qu'en 1975, initiée par le programme électronucléaire thodanien (C.T.G.R.E.F., 1976, 1977). Divers travaux (ROUX, 1984 ; BRAVARD, 1987 ; PATTEE, 1988 ; ROUX et al., 1989 ; FRUGET, 1992) et rapports de synthèse (AGENCE DE BASSIN R.M.C., 1988 ; DELEGATION DE BASSIN R.M.C., 1992) exposent la multiplicité des actions anthropiques à l'origine des altérations de l'hydrosystème.

Les chroniques obtenues à ce jour grâce aux suivis hydrobiologiques des sites aménagés permettent une approche "dynamique" de l'ichtyocénose contemporaine. PERSAT (1988) a ainsi montré les modifications structurales de l'ichtyocénose du Haut-Rhône entre 1978 et 1986, consécutives à l'aménagement du fleuve. Un suivi, réalisé entre 1982 et 1992 sur un aménagement du tiers amont du Bas-Rhône mis en service en 1977, a montré la relative stabilité des populations d'un peuplement peu diversifié (OLIVIER et al., 1994).

Ce travail s'intègre dans cette perception à moyen terme, de 1983 à 1994, de l'évolution du peuplement piscicole. II porte sur le tronçon médian du Bas-Rhône, aménagé à partir de 1952 par la Compagnie Nationale du Rhône. II s'intéresse, en particulier, à l'influence de la variabilité hydrologique sur la composition des communautés.

\section{MATÉRIEL ET MÉTHODES}

Le tiers central du Bas-Rhône s'étend depuis le confluent de l'Isère jusqu'au confluent de l'Ardèche. II comprend cinq aménagements hydro-électriques construits entre 1948 et 1968. Deux centrales nucléaires (fig.1) ont été construites sur les deux aménagements aval de ce secteur. La centrale de Tricastin (1980-1981) est implantée sur le canal d'amenée de l'aménagement de Donzère-Mondragon. La centrale de CruasMeysse (1984-1986) se trouve sur la retenue de l'aménagement de Montélimar.

Trois stations prospectées depuis 1983 , entre la restitution de l'usine de Baix-Logis Neuf et celle de Bollène, ont été retenues compte tenu:

- de leur valeur descriptive du chenal aménagé,

- de leurs caractéristiques physiques inchangées depuis la construction des barrages,

- de l'absence d'effets directs liés aux stations de pompage et aux rejets thermiques des centrales nucléaires.

L'échantillonnage est réalisé par pêche électrique depuis une embarcation selon le protocole proposé par ALLARDI et al. (1975). La pêche est exercée en continu le long de la rive.

Bien que chaque station fasse l'objet de plusieurs pêches par an, une seule pêche par année et par station a été prise en compte : celle pratiquée au printemps lorsque la température des eaux est supérieure à $8^{\circ} \mathrm{C}$. Sur ce tronçon de $40 \mathrm{~km}$, les stations ont été considérées représentatives de 3 faciès de rives du cours principal aménagé (chenal endigué, retenue, canal d'amenée). Les résultats des 3 pêches ont été regroupés après correction selon l'effort de pêche (20 minutes de prospection). La discussion porte sur des effectifs moyens. 

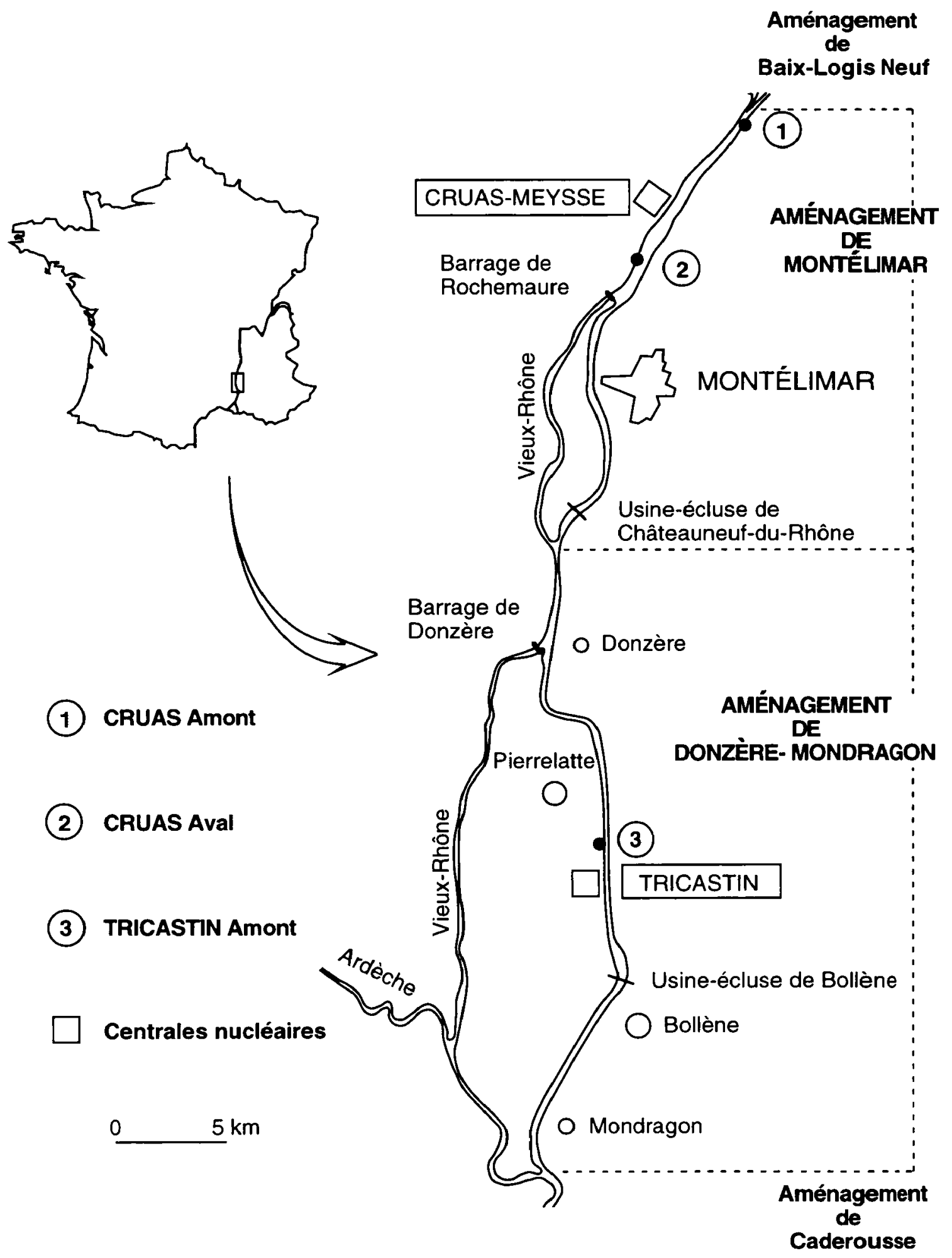

(1) CRUAS Amont

(2) CRUAS Aval

(3) TRICASTIN Amont

Figure 1 : Bas-Rhône. Localisation du secteur fluvial étudié et des trois stations de pêche.

Figure 1 : Lower Rhône River. Investigation area and location of three sampling sites. 
La structure du tableau obtenu a été étudiée par une Analyse Factorielle des Correspondances à partir du logiciel ADE (CHESSEL et DOLEDEC. 1992).

\section{RÉSULTATS}

\section{Généralités}

A I'heure actuelle, le peuplement de ce secteur compte 33 espèces appartenant à 12 familles (Tableau I).

Le Bas-Rhône présente un fond faunistique réduit, constitué de 5 espèces euryoeciques (Alburnus alburnus, Rutilus rutilus, Leuciscus cephalus, Anguilla anguilla et Lepomis gibbosus) dont les occurrences sont supérieures à $60 \%$. Ces 5 espèces représentent $92 \%$ des effectifs.

Des espèces appartenant au peuplement originel ( $\left.{ }^{*}\right)$ ont disparu de ce secteur (Alosa sp., Petromyzon marinus), d'autres sont devenues rares (Leuciscus leuciscus, Phoxinus phoxinus, Lota lota, Cottus gobio) ou peu abondantes (Leuciscus soufia, Chondrostoma toxostoma, Esox lucius).

Indépendamment des 6 espèces apparues au siècle dernier, 3 nouvelles espèces ont été capturées au cours de cette décennie: Silurus glanis, Pseudorasbora parva et Carassius auratus.

Le silure, introduit à la fin des années soixante sur la Seille (Saône-et-Loire), a progressivement colonisé la Saône puis le Rhône. Le pseudorasbora a colonisé le bassin du Rhône par l'entremise de la pisciculture (ALLARDI et CHANCEREL, 1988). Cette espèce est désormais bien représentée sur le tiers aval du Bas-Rhône (NICOLAS et PONT, 1995). Le carassin doré a usé de voies similaires. II a bénéficié des conditions thermiques plus élevées de ces dernières années. II pourrait s'agir de Carassius auratus gibelio, espèce ayant envahi le Danube médian et inférieur (HOLCIK, 1991).

\section{Evolution du peuplement et variabilité interannuelle}

Les deux premiers axes factoriels de l'Analyse Factorielle des Correspondances du Tableau I, après élimination de 5 espèces capturées une seule fois (Carassius auratus, Phoxinus phoxinus, Silurus glanis, Micropterus salmoïdes et Cottus gobio), présentent les oscillations successives de la structure du peuplement autour du noyau central formé des espèces dominantes (fig. 2). Il est possible de distinguer 3 périodes : 1983-1984, 1986-1988 et 1990-1993. Les modifications les plus remarquables s'effectuent en 1985, 1989 et 1994.

Les années 1983 et 1984 sont essentiellement marquées par l'abondance de l'anguille, la deuxième période (1985-1988) par la prédominance de l'ablette, notamment en 1987 et 1988. La période 1990-1993 est caractérisée par l'abondance du gardon, consécutive à la fraye exceptionnelle de 1989. Les relevés de 1994 montrent la réapparition des espèces rhéophiles, la capture du chabot et du vairon, une chute des effectifs de gardons.

La structure faunistique se déforme:

- soit par dominance des espèces les plus tolérantes vis-à-vis des caractères eutrophes et lénitiques du milieu (gardon, brème bordelière) et une réduction du nombre d'espèces (années 1991 et 1992),

- soit par la dominance du chevaine et une augmentation générale du nombre d'espèces (années 1989 et 1994).

(*) Ce peuplement, décrit par KREITMANN (1932) et LEGER (1945), comprenait un plus grand nombre d'espèces et était caractérise par l'existence

- d'un groupe d'espèces dominantes constitué par le hotu. le chevaine, le goujon, la brème commune, le brochet, l'alose feinte,

- d'un groupe d'espèces communes comprenant le barbeau, la vandoise, le vairon. l'ablette, la perche, l'anguille, la lamproie marine. 
Tableau I: Liste des espèces capturées sur le secteur de Cruas-Tricastin. Occurrences (Oc \%) sur la base de 248 pêches électriques réalisées entre 1980 et 1994 sur 6 stations. Effectifs corrigés moyens (nombre d'individus pour $20 \mathrm{mn}$ de pêche) calculés à partir des pêches réalisées au printemps de chaque année dans les 3 stations étudiées.

Table I : Occurrences (in percentages) of 33 species in the Cruas-Tricastin sector, based on 248 electrofishing samples collected from 1980 to 1994 in 6 stations. Corrected mean abundances (catch per unit effort of 20 minutes) obtained every spring in the 3 stations.

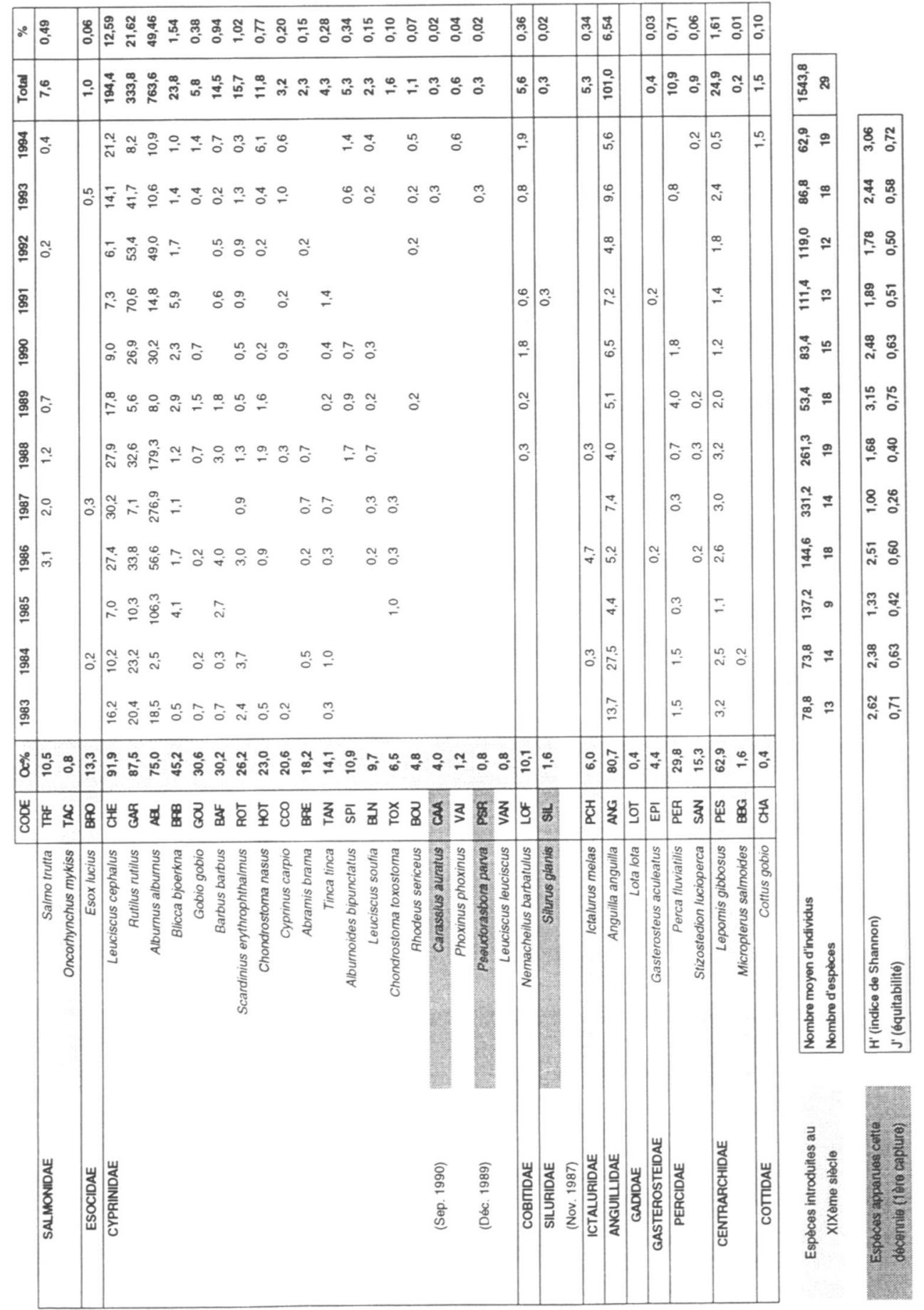


1

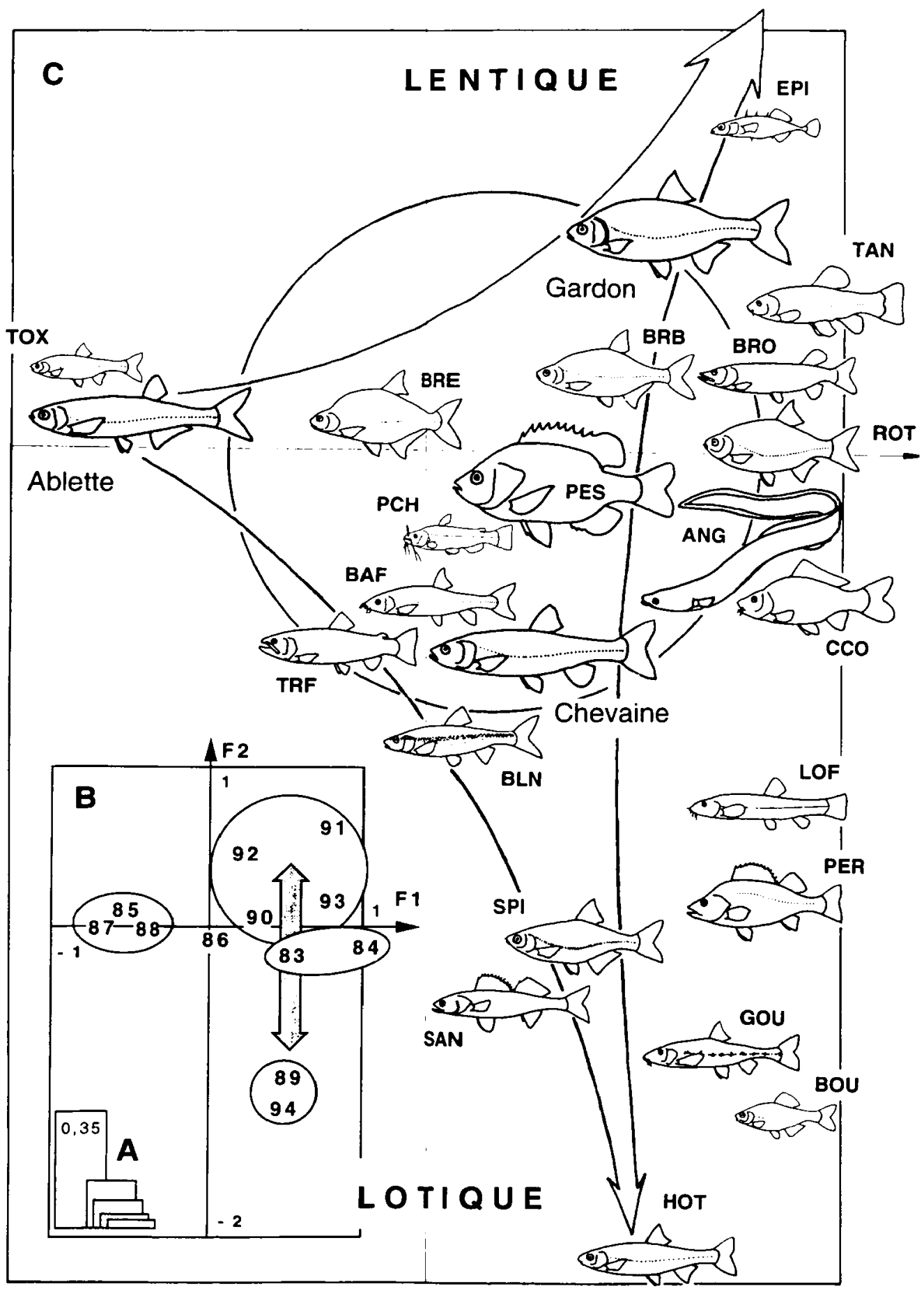

Figure 2 : Représentation graphique de l'Analyse Factorielle des Correspondances. A. Histogramme des valeurs propres. B. Carte F1-F2 des années. C. Carte F1-F2 des espèces.

Figure 2 : Ordination of species and years by correspondence analysis. A. Histogram of eigenvalues. B. Distribution of years on the F1 x F2 map. C. Species distribution on the F1 $\times$ F2 map. 
Le peuplement retrouve par épisodes une structure spécifique altérée du type originel. Mais les facteurs responsables de cette restructuration ne peuvent déstabiliser durablement la hiérarchie établie par les espèces euryoeciques.

Bien que des fluctuations cycliques des effectifs existent chez la plupart des espèces, seules seront considérées les espèces à forte occurrence.

Le coefficient de variation (CV), utilisé pour quantifier la stabilité des populations (GROSSMAN et al.,1990), a été calculé pour les 5 espèces ubiquistes. Selon le critère de classification proposé par FREEMAN et al. (1988), les 5 populations se répartissent sensiblement dans les 4 catégories proposées : (1) CV $\leq 25 \%=$ très stable, (2) $26 \% \leq \mathrm{CV}$ $\leq 50 \%=$ peu stable, (3) $51 \% \leq \mathrm{CV} \leq 75 \%=$ fluctuante, (4) $\mathrm{CV} \geq 76 \%=$ très fluctuante.

\section{Espèces}

Anguilla anguilla (de 1986 à 1994)

Lepomis gibbosus

Leuciscus cephalus

Rutilus rutilus

Alburnus alburnus

\begin{tabular}{c} 
CV $(\%)$ \\
\hline 29 \\
43 \\
54 \\
72 \\
133
\end{tabular}

population stable population peu stable population fluctuante population fluctuante population très fluctuante

Ces fluctuations sont partiellement expliquées par les conditions hydrologiques et thermiques de la période considérée (fig. 3). L'hydraulicité a été forte avant 1989, puis faible jusqu'à la fin 1993. La température moyenne annuelle des eaux a baissé de 1982 à 1984, s'est stabilisée de 1985 à 1987, puis a réaugmenté de 1988 à 1994. Les faibles débits de 1989, et une température estivale élevée, ont été propices au recrutement des espèces lénitophiles et thermophiles. Des conditions hydroclimatiques similaires de 1990 à 1992, ont permis une bonne survie des cohortes apparues en 1989, mais le recrutement a été modeste. Les crues de l'automne 1993 et de janvier 1994 ont restructuré le milieu et les peuplements. Les effets, encore peu perceptibles au printemps 1994, ont été remarqués lors de la fraye (fort recrutement de l'ablette et de diverses espèces lithophiles).

\section{DISCUSSION}

A l'échelle du tronçon fluvial étudié, les oscillations cycliques des effectifs et les valeurs du CV montrent que le mode de régulation des populations n'est pas purement stochastique. Si les conditions hydroclimatiques contrôlent le niveau supérieur de la variabilité interannuelle, d'autres facteurs abiotiques (tels que l'habitat) et biotiques (stratégie écologique des espèces, relations intra- ou inter-spécifiques) ont un rôle indéniable.

\section{Le contrôle hydroclimatique}

Les fluctuations d'abondance des espèces sont essentiellement contrôlées par la température et l'hydrologie (LINFIELD, 1985). MILLS et MANN (1985) ont montré que seules certaines années permettent l'obtention de larges cohortes : l'année 1989 est caractéristique de ce point de vue. L'abondance du gardon de 1990 à 1993 n'est que le reflet de l'importance de cette classe d'âge.

L'hydrologie agit à de multiples niveaux. Lorsque les débits augmentent et dépassent la capacité des usines, les crues empruntent les anciens tracés du Rhône court-circuités par les dérivations. Selon la période, l'amplitude et la durée de la crue, elles favorisent ou perturbent la fraie dans ces annexes fluviales. Elles entraînent la dispersion des larves et des juvéniles, et renouvellent ainsi le peuplement du chenal principal (COPP et al., 1991).

\section{Les facteurs biotiques}

La variabilité observée est fonction des caractéristiques biologiques de chaque espèce, et de la stratégie adoptée dans l'exploitation des ressources disponibles. 


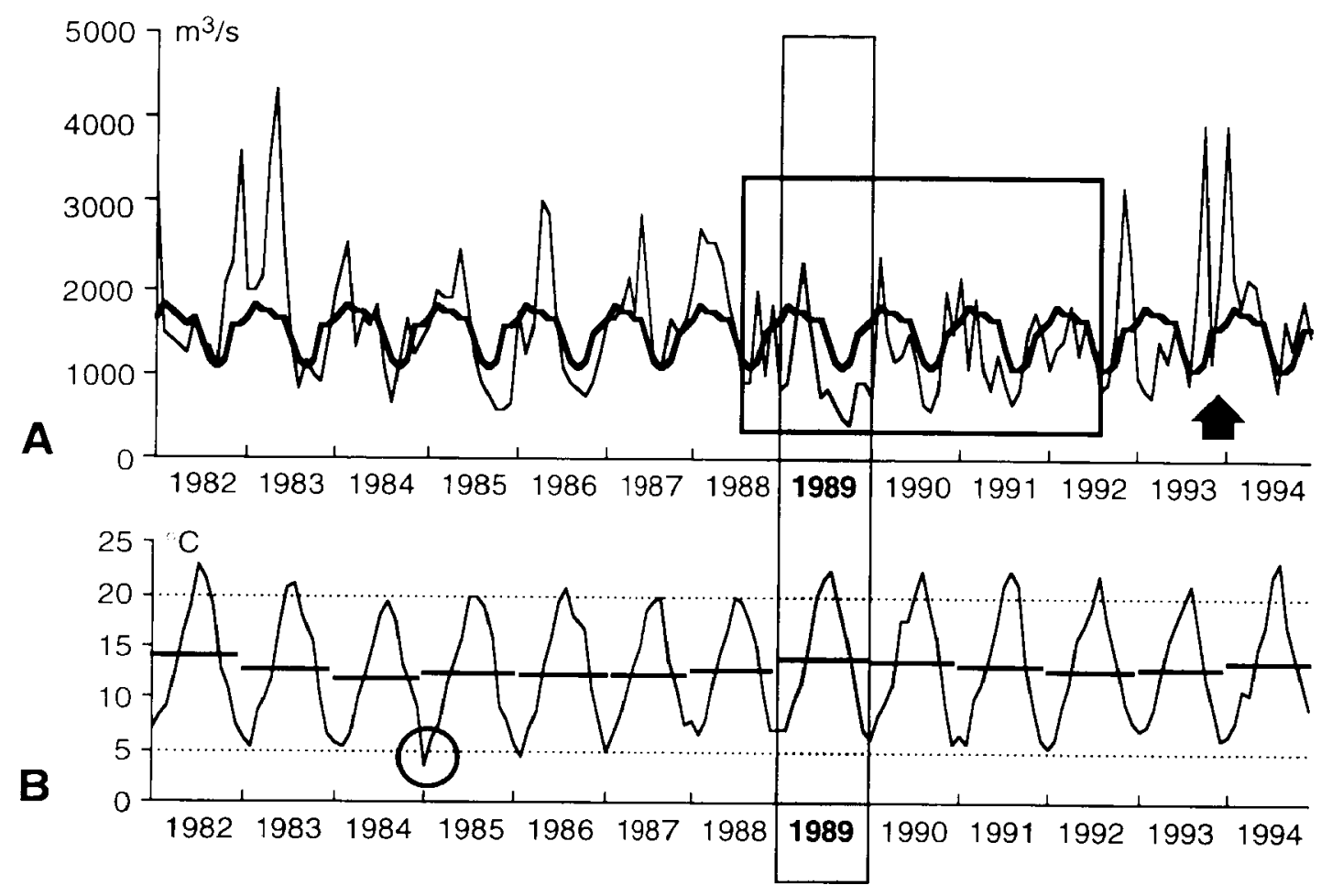

Figure 3 : A. Débits moyens mensuels du fleuve à Viviers entre 1982 et 1994 . Le trait épais donne le débit mensuel de référence (période 1920-1993). Données de la Compagnie Nationale du Rhône. B. Température moyenne mensuelle des eaux en amont de la centrale nucléaire de Tricastin. Le trait horizontal donne la moyenne annuelle. Données fournies par Electricité de France.

Figure 3 : A. Average monthly discharges of the Lower Rhône at Viviers from 1982 to 1994. The thick line represents reference discharge over the period 19201993. Data provided by Compagnie Nationale du Rhône. B. Average monthly temperature of the Rhône upstream from Tricastin nuclear power plant. The thick horizontal line represents the annual mean. Data provided by Electricité de France.

Les effectifs de l'anguille, espèce ayant une répartition homogène dans les faciès à enrochements, sont peu fluctuants (de 1986 à 1994).

Une espèce longévive comme le chevaine, capturable en rive quelle que soit sa taille, présente des fluctuations d'effectifs pondérées sur plusieurs années. La distribution est distincte de celle à forte amplitude et de courte durée observée chez l'ablette, espèce peu longévive, grégaire et pélagique.

L'alternance des années à fort puis faible recrutement par le jeu de la compétition intraspécifique, signalée chez le gardon dans des systèmes eutrophes lacustres (TOWNSEND et PERROW, 1989), est probable en système fluvial aménagé malgré le puissant contrôle des facteurs hydroclimatiques. Cette double influence des paramètres abiotiques et biotiques sur le recrutement du gardon a été constatée sur le tiers aval (POIZAT, 1993) et le tiers amont du Bas-Rhône (OLIVIER et al., 1994).

La stratégie de reproduction peut minimiser la variabilité : c'est le cas de la perche soleil qui protège sa progéniture. 


\section{Les actions anthropiques}

Les effectifs de l'anguille ont chuté en 1985. Deux explications sont possibles

- une baisse significative et pluriannuelle consécutive aux échantillonnages répétés sur les mêmes stations, problème évoqué par LOBON-CERVIA et al. (1990):

- les conséquences d'une pollution accidentelle par des produits phytosanitaires survenue au printemps 1985 sur le tiers amont du Bas-Rhône (AGENCE DE BASSIN, 1988).

Cette dernière hypothèse pourrait justifier la faible diversité enregistrée cette année.

\section{CONCLUSIONS}

Le chenal du Bas-Rhône présente de grandes similarités avec les autres fleuves européens tels que le Danube, le Rhin ou la Vistule, aménagés à des fins énergétiques et de navigation [BACLABASA-DOBROVICI (1989), BACKIEL et PENCZAK (1989), LELEK (1989), SCHIEMER et SPINDLER (1989)]. Les Cyprinidae dominants sont les mêmes. Le cortège d'espèces d'accompagnement dépend du contexte biogéographique, de la localisation de la station dans la structure aménagée et de sa proximité avec les annexes fluviales disponibles dans la plaine alluviale: bras latéraux, affluents, sections court-circuitées.

Bien que la technique de pêche ne puisse donner une image parfaite de l'ichtyocénose, les assemblages piscicoles de bordure constituent de bons indicateurs de l'état général de la communauté (BAIN, 1982). Ces habitats abritent la majorité des espèces au cours de la période juvénile. Lors d'un aménagement du fleuve, les principaux impacts concernent la complexité de cette interface "mobile" (JUNK et al., 1989) entre les milieux aquatiques et terrestres (SCHLOSSER, 1991). Si les conséquences sur les populations et la dynamique des communautés piscicoles peuvent être perçues par le biais de cet indicateur sensible qu'est l'assemblage piscicole des rives, la diversité des facteurs responsables de la variabilité interannuelle exige des études à long terme pour une appréciation correcte des évolutions temporelles du peuplement.

\section{REMERCIEMENTS}

Les données piscicoles utilisées dans ce travail proviennent de conventions d'étude établies entre le CEMAGREF et Electricité de France. Nous remercions la direction d'EDF et les responsables des sites nucléaires de Cruas-Meysse et de Tricastin de nous avoir aimablement accordé l'usage de ces données.

\section{BIBLIOGRAPHIE}

AGENCE DE BASSIN Rhône-Méditerranée-Corse, 1988. Qualité du fleuve Rhône. Synthèse des connaissances. Agence de l'Eau Rhône-Méditerranée-Corse, Lyon, $251 \mathrm{p}$.

ALLARDI J., CHANCEREL F., 1988. Note ichtyologique sur la présence de Pseudorasbora parva (Schlegel, 1842). Bull. Fr. Pêche Piscic., 308, 35-37.

ALLARDI J., DUGUET C., LEYNAUD G., 1975. Etude du peuplement ichtyologique d'un milieu fluvial réchauffé artificiellement. In WELCOMME R.L. (ed.), Symposium sur les méthodes de prospection, de surveillance et d'évaluation des ressources ichtyologiques dans les lacs et grands cours d'eau, 209-227, FAO, EIFAC Techn. Paper 23 (Suppl.1).

BACKIEL T., PENCZAK T., 1989. The fish and fisheries in the Vistula River and its tributary, the Pilica River. In DODGE D.P. (ed.), Proceedings of the International Large River Symposium. Can. Spec. Publ. Fish. Aquat. Sci., 106, 488-503.

BACLABASA-DOBROVICI N., 1989. The Danube River and its fisheries. In DODGE D.P. (ed.), Proceedings of the International Large River Symposium. Can. Spec. Publ. Fish. Aquat. Sci., 106, 455-468.

BAIN M.B., 1982. Study designs and sampling techniques for community-level assessment of large rivers. In CUFFNEY T.F., GURTZ M.E., Biological assessments in large rivers, 63-74, North American Benthological Society, Louisville, Kentucky. 
BRAVARD J.P., 1987. Le Rhône, du Léman à Lyon. La Manufacture, Lyon, 451 p.

CHESSEL D., DOLEDEC S., 1992. ADE Software. Multivariate Analyses and Graphical Display for Environmental Data (version 3.4). Vol. 1. User's manual (in English), Vol. 2. Examples (in French), Vol. 3. Graphics Documentation (in French). Université Lyon I, $523 \mathrm{p}$.

COPP G.H., OLIVIER J.M., PENAZ M., ROUX A.L., 1991. Juvenile fishes as functional describers of fluvial ecosystem dynamics : applications on the river Rhône, France. Regul. Riv. : Research \& Management, 6, 135-145.

C.T.G.R.E.F., 1976. Etude écologique des sites d'implantation des centrales électriques sur le Rhône. Première partie : caractéristiques mésologiques. Deuxième partie : les peuplements. Division QEPP, Groupement d'Antony, Rapport EDF, 49 \& 33 p.

C.T.G.R.E.F., 1977. Etude écologique du Rhône. Sites de Bugey, Chavanay et Tricastin. Etat de référence. Divisions QEPP, Groupements d'Antony et d'Aix-en-Provence, Rapport EDF, $102 \mathrm{p}$.

DELEGATION DE BASSIN Rhône-Méditerranée-Corse, 1992. Schéma de vocation piscicole du fleuve Rhône. Rapport de synthèse. 202 p. + annexes.

FREEMAN M.C., CRAWFORD M.K., BARRETT J.C., FACEY D.E., FLOOD M.G., HILL J., STOUDER D.J., GROSSMAN G.D., 1988. Fish assemblage stability in a southern Appalachian stream. Can. J. Fish. Aquat. Sci., 45, 1949-1958.

FRUGET J.F., 1992. Ecology of the lower Rhône after 200 years of human influence : a review. Regul. Riv. : Research \& Management, 7, 233-246.

GROSSMAN G.D., DOWD J.F., CRAWFORD M.K., 1990. Assemblage stability in stream fishes : a review. Environ. Manage., 14, 661-671.

HOLCIK J., 1991. Fish introductions in Europe with particular reference to its central and eastern part. Can. J. Fish. Aquat. Sci., 48 (Suppl. 1), 13-23.

JUNK W.J., BAYLEY P.B., SPARKS R.E., 1989. The flood pulse concept in river-floodplain systems. In DODGE D.P. (ed.), Proceedings of the International Large River Symposium. Can. Spec. Publ. Fish. Aquat. Sci., 106, 110-127.

KREITMANN L., 1932. Les grandes lignes de l'économie piscicole du bassin français du Rhône. Trav. Lab. Hydrobiol. Pisc. Univ. Grenoble, 24, 127-132.

LEGER L., 1945. Etude sur l'hydrobiologie et l'économie piscicoles du département du Rhône (avec une carte et un graphique). Trav. Lab. Hydrobiol. Pisc. Univ. Grenoble, 37, 1-14.

LELEK A., 1989. The Rhine River and some of its tributaries under human impact in the last two centuries. In DODGE D.P. (ed.), Proceedings of the International Large River Symposium. Can. Spec. Publ. Fish. Aquat. Sci., 106, 469-487.

LINFIELD R.S.J., 1985. An alternative concept to home range theory with respect to populations of cyprinids in major river systems. J. Fish Biol., 27 (Suppl. A), 187-196.

LOBON-CERVIA J., BERNAT Y., RINCON P.A., 1990. Effects of eel (Anguilla anguilla L.) removals from selected sites of a stream on its subsequent densities. Hydrobiologia, 206, 207-216.

MILLS C.A., MANN R.H.K., 1985. Environmentally-induced fluctuations in year-class strength and their implications for management. J. Fish Biol., 27 (Suppl. A), 209-226.

NICOLAS Y., PONT D., 1995. Importance d'annexes latérales artificielles pour le recrutement en juvéniles de poissons dans un fleuve aménagé, le Bas-Rhône. Bull. Fr. Pêche Piscic., 337/338/339.

OLIVIER J.M., CENTOFANTI M., FRUGET J.F., PERSAT H., BOISSON C., 1994. Characterization and evolution of the fish community of the regulated middle Rhone river : results of a 10 years survey period. Communication, Sixth International Symposium on Regulated Streams, Ceske Budejovice, Czech Rep. 
PATTEE E., 1988. Fish and their environment in large European river ecosystems. The Rhône. Rev. Sci. Eau, 7, 35-74.

PERSAT H., 1988. De la biologie des populations de l'Ombre commun Thymallus thymallus (L. 1758) à la dynamique des communautés dans un hydrosystème fluvial aménagé, le Haut-Rhône français. Thèse de Doctorat d'Etat, Univ. Cl. Bernard Lyon 1, $223 p$.

POIZAT G., 1993. Echelle d'observation et variabilité des abondances de juvéniles de poissons dans un secteur aval du Rhône. Thèse de Doctorat, Univ. Cl. Bernard Lyon 1, $217 \mathrm{p}$.

ROUX A.L., 1984. The impact of emptying and cleaning reservoirs on the physico-chemical and biological water quality of the Rhône downstream of the dams. In LILLEHAMMER A., SALTVEIT S.J. (Eds), Regulated rivers, 61-70, Universitetsforlaget, Oslo.

ROUX A.L., BRAVARD J.P., AMOROS C., PAUTOU G., 1989. Ecological changes of the French Upper Rhône River since 1750. In PETTS G.E., MOLLER H., ROUX A.L. (eds), Historical change of large alluvial rivers : Western Europe, 323-350, John Wiley \& Sons, Chichester.

SCHIEMER F., SPINDLER T., 1989. Endangered fish species of the Danube River in Austria. Regul. Riv. : Research \& Management, 4, 397-407.

SCHLOSSER I.J., 1991. Stream fish ecology : a landscape perspective. BioScience, 41, 704-712.

TOWNSEND C.R., PERROW M.R., 1989. Eutrophication may produce population cycles in roach, Rutilus rutilus (L.), by two contrasting mechanisms. J. Fish Biol., 34, 161-164. 